\title{
Experimental test of the Pauli Exclusion Principle
}

\author{
A S Barabash \\ Institute of Theoretical and Experimental Physics, B. Cheremushkinskaya 25, 117218 \\ Moscow, Russia \\ E-mail: barabash@itep.ru
}

\begin{abstract}
.
A short review is given of three experimental works on tests of the Pauli Exclusion Principle (PEP) in which the author has been involved during the last 10 years. In the first work a search for anomalous carbon atoms was done and a limit on the existence of such atoms was determined, ${ }^{12} \tilde{\mathrm{C}} /{ }^{12} \mathrm{C}<2.5 \times 10^{-12}$. In the second work PEP was tested with the NEMO-2 detector and the limits on the violation of PEP for p-shell nucleons in ${ }^{12} \mathrm{C}$ were obtained. Specifically, transitions to the fully occupied $1 s_{1 / 2}$-shell yielded a limit of $4.2 \times 10^{24} \mathrm{y}$ for the process with the emission of a $\gamma$-quantum. Similarly limits of $3.1 \times 10^{24} \mathrm{y}$ for $\beta^{-}$and $2.6 \times 10^{24}$ y for $\beta^{+}$Pauli-forbidded transition of ${ }^{12} \mathrm{C} \rightarrow{ }^{12} \tilde{\mathrm{N}}\left({ }^{12} \tilde{\mathrm{B}}\right)$ are reported. In the third work it was assumed that PEP is violated for neutrinos, and thus, neutrinos obey at least partly the Bose-Einstein statistics. Consequences of the violation of the exclusion principle for double beta decays were considered. This violation strongly changes the rates of the decays and modifies the energy and angular distributions of the emitted electrons. It was shown that pure bosonic neutrinos are excluded by the present experimental data. In the case of partly bosonic neutrinos the analysis of the existing data allows one to put an upper bound for $\sin ^{2} \chi<0.6$. The sensitivity of future measurements is also evaluated.
\end{abstract}

PACS numbers: 23.40.-s, $14.80 . \mathrm{Mz}$ 


\section{Introduction}

The Exclusion Principle is one of the most fundamental laws of nature. It was formulated by W. Pauli in 1925 [1] to explain the regularities of the Periodic Table of elements and the characteristic features of atomic spectra. In modern Quantum Field Theory (QFT) the Exclusion Principle appears automatically from the nature of identical particles and the anti-commutativity of the fermion creation (annihilation) operators. It postulates that in a system of identical fermions, two or more particles cannot occupy the same state.

The discovery in 1956 of parity non-conservation in $\beta$-decay [2] showed for the first time that "fundamental laws" can be violated. The violation of CP invariance was then discovered in 1964 [3]. As a result, all conservation laws began to be tested. Some of them, for example, the non-conservation of leptonic and barionic quantum numbers can be explained in the framework of models satisfying all the principles of standard QFT. Others, such as the non-conservation of the electric charge [4, 5, 6, 7], CPT-violation [8, 9], and Lorentz-invariance violation [10, 11, require a global reconstruction of modern theoretical physics to create self-consistent models.

In more recent publications [12, 13, 14, 15, 16, 17, some attempts were made to introduce into the theory a small violation of PEP, but they have not been successful. PEP is at the heart of the QFT and its violation, even if very small, leads to the appearance of states with negative norma (negative probability) [18, 19]. Thus there is no answer to the question, "What is the accuracy of PEP?". The reason for this is that there is no real self-consistent and non-contradictory model, with small PEP violation. Indeed any model with PEP violation must be beyond the standard QFT. It was L.B. Okun [17, 13], who said," That exceptional place occupied by the Pauli principle in modern physics does not imply that it does not need further painstaking experimental tests. Quite the opposite: the fundamental character of this principle generates special interest to its quantitative testing throughout the Mendeleev Table".

Experimental searches of the effects of the Pauli principle violation via electrons (see, for example, [20, 21, 22, 23, 24] and review [25]) and nucleons (see, for example, [26, 27, 28, 29, 30, 31]) which have given negative results, leading to extremely strong bounds on the magnitude of the violation.

In this paper results obtained in Ref. [20], [26], and [32] are presented.

\section{Search for anamalous carbon atoms is sought as evidence of the violation of PEP during the period of nucleosynthesis [20]}

This paper addresses a search for anomalous ("non-Paulian") atoms. Such atoms could be of cosmological origin, if not all $10^{80}$ electrons in the universe are antisymmetrized or if spontaneous transitions of ordinary atoms into "non-Paulian" atoms are nevertheless possible. The chemical properties of atoms with three electrons per 1 s shell must be similar to the properties of their "lower-order" neighbors in the periodic table (for 
example, "non-Paulian" carbon would be similar to boron).

In 1989 Novikov and Pomansky proposed a check of PEP by searching for anomalous atoms arising from the periods of nucleosynthesis [33]. If PEP is violated, then every substance containing elements with the atomic number $\mathrm{Z}$ contains an admixture of anomalous atoms of the element with the atomic number $(Z+1)$, since these anomalous atoms have the same chemical properties as the element with the atomic number $Z$. The concentration of anomalous atoms in a substance is high in the case that the cosmic abundance of the parent element $(Z+1)$ is high, while that of the element $\mathrm{Z}$ is low. If the formation of "non-Paulian" atoms occurred as a result of a spontaneous transition of an outer electron into an inner shell, then the concentration of anomalous atoms in the material will be

$$
C=t \times P(Z+1) / \tau \times P(Z),
$$

where $t$ is the average time which has passed since the moment when the anomalous atom formed to the end of presolar system formation $\left(\sim 4.5 \times 10^{9} \mathrm{y}[33]\right) ; \tau$ is the lifetime of an atomic electron with respect to the violation of PEP; and, $\mathrm{P}(\mathrm{Z}+1)$ and $\mathrm{P}(\mathrm{Z})$ are the cosmic abundances of elements with atomic numbers $(Z+1)$ and $Z$.

In Ref. [33] two pairs of atoms were proposed as the most promising for the investigation: boron-carbon and fluorine-neon, with the ratios $P(Z+1) / P(Z)=$ $2.18 \times 10^{6}$ and 650 , respectively. An experimental search for anomalous neon atoms in fluorine and argon atoms in chlorine has been performed [21] (the results obtained by accelerator mass spectrometry are $\tau>2 \times 10^{30}$ y and $\tau>4 \times 10^{27}$ y), while the boron-carbon pair remained unstudied.

The work [20] was devoted to the search for anomalous carbon atoms in boron. Recall that boron exists in the form of two stable isotopes, ${ }^{10} \mathrm{~B}(\sim 19 \%)$ and ${ }^{11} \mathrm{~B}(\sim$ $81 \%)$. Carbon likewise consists of two stable isotopes, ${ }^{12} \mathrm{C}(\sim 99 \%)$ and ${ }^{13} \mathrm{C}(\sim 1 \%)$. The anomalous atom ${ }^{12} \tilde{\mathrm{C}}$ contains three $\mathrm{K}$-shell electrons and therefore behaves chemically like a boron atom. Such anomalous atoms should be concentrated in boron and its compounds in the process of evolution. In order to observe them a search must be conducted for anomalous ${ }^{12} \mathrm{C}$ nuclei in boron or for boron atoms with a nuclear mass of 12 .

In the present work the first possibility was investigated. In discussing possible experiments in search of anomalous carbon atoms in boron, Novikov and Pomansky proposed mass spectrometry and even estimated the sensitivity of experiments of this kind. However, their estimate is much too high, because of a large admixture of "ordinary" carbon (at the $0.1 \%$ level) is always present in boron samples and in the residual atmosphere of the mass spectrometer.

The idea of this experiment was to remove carbon atoms from a boron sample and then to measure the content of carbon nuclei in it. The search for anomalous carbon atoms was conducted by $\gamma$-activation analysis of different boron samples. Boron is ideal for the $\gamma$-activation analysis, since irradiation with $\gamma$-quanta does not produce radioactive isotopes and the activity is thereby determined by the impurities. 
The experiment was performed on the microtron at the Institute of Physics Problems of the Russian Academy of Sciences. The boron samples for analysis consisted of amorphous boron (powder, obtained from $\mathrm{BCl}_{3}$ gas), $\alpha$ - and $\beta$-rhombohedral boron, boron whiskers on tungsten, decaborane $\left(\mathrm{B}_{10} \mathrm{H}_{14}\right)$, and boron grown by zone melting. Experiments showed that the purest boron was obtained by zone melting, and boron from this source was used for the most conclusive measurements. In these samples the initial content of carbon atoms was equal to approximately $1.5 \times 10^{-3} \mathrm{~g} / \mathrm{g}$. For this reason, it is impossible to search for anomalous carbon atoms in boron by the method of nondestructive $\gamma$-spectrometry on irradiated samples, and another special method was developed for removing impurities from the boron.

A boron sample with a mass of $\sim 0.1 \mathrm{~g}$ was irradiated with $\gamma$-quantum bremsstraglung from $28-\mathrm{MeV}$ electrons. If ${ }^{12} \mathrm{C}$ nuclei are present in the boron, then the reaction ${ }^{12} \mathrm{C}(\gamma, \mathrm{n}){ }^{11} \mathrm{C}$ should occur, i.e., radioactive ${ }^{11} \mathrm{C}$ nuclei with a half-life of $20.34 \mathrm{~min}$ are formed. In $99 \%$ of the cases ${ }^{11} \mathrm{C}$ undergoes $\beta^{+}$decay and can therefore be detected by the $\gamma-\gamma$ coincidence method (the detection of two $511 \mathrm{keV}$ annihilation $\gamma$-rays, which are emitted after the positrons stop and are annihilated in the material).

After irradiation, the boron sample was oxidized in a $\mathrm{KNO}_{3}-\mathrm{KOH}(3: 1)$ melt for about $10 \mathrm{~min}$ at $600{ }^{\circ} \mathrm{C}$. In the process of oxidative melting in an alkaline melt, the carbon-containing impurities in boron (a solid solution of carbon, i.e., boron carbide $\mathrm{B}_{4} \mathrm{C}$ ) are oxidized to $\mathrm{CO}_{2}, \mathrm{CO}, \mathrm{KHCO}_{3}$, and $\mathrm{K}_{2} \mathrm{CO}_{3}$, while boron is oxidized to $\mathrm{B}_{2} \mathrm{O}_{3}$. The cooled melt was then dissolved in water containing nitric acid to convert the boron anhydride into boric acid $\mathrm{H}_{3} \mathrm{BO}_{3}$. Next, the solution was heated to the boiling point. In this process the potassium bicarbonates and carbonates decomposed, and carbon dioxide gas was released. The carbon dioxide was removed from the boric acid solution by a flow of air. Experiments on measuring the activity of the released ${ }^{11} \mathrm{CO}_{2}$ showed that in 25 min such a procedure removes more than $99 \%$ of the carbon from the solution. After the $\mathrm{CO}_{2}$ was driven off, the change in the activity of ${ }^{11} \mathrm{C}$ nuclei remaining in the boric solution was measured as a function of time. Graphite was irradiated simultaneously with the sample. Measurements of the activity of the graphite made it possible to calculate the mass of the remaining carbon.

The plot displayed in Fig. 1 shows the results of one of these measurements. The residual activity is due essentially to the background which remains at approximately 40 decays per $100 \mathrm{~s}$ and only a slight elevation in activity at the beginning of the measurements can be attributed to radioactive carbon nuclei with 20.34-min half-life. The inset in the figure displays on a large scale the result of the measurements of the activity of the solution and the result of the mathematical analysis of the data using the function $a+b \times \exp \left(-t / T_{1 / 2}\right)$, where $a$ is the constant background and $T_{1 / 2}=1224$ $\mathrm{s}$ is the half-life of ${ }^{11} \mathrm{C}$.

A series of measurements of this kind established that the concentration of anomalous carbon atoms does not exceed $5 \times 10^{-6} \mathrm{~g} / \mathrm{g}$. The point is that one cannot rule out the possibility that the observed activity of ${ }^{11} \mathrm{C}$ nuclei is due to the residual content of "normal" carbon in the boron sample. For this reason, it can only be asserted 


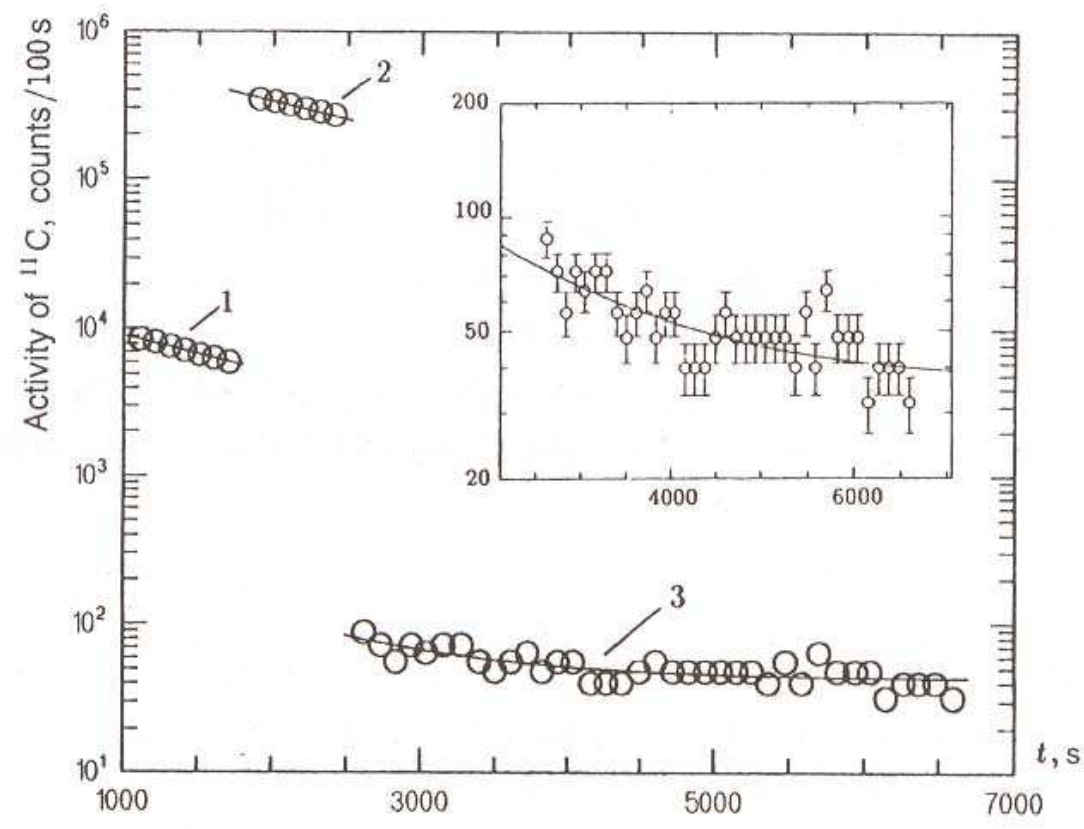

Figure 1. Time dependence of the positron activity of carbon separated from a 100 mg boron sample after irradiation (1), a $6 \mathrm{mg}$ sample irradiated together with the experimental sample (2), and a solution after chemical separation of carbon atoms (3). Inset: the detected positron activity of the solution. The solid line is a fit of the data assuming that only ${ }^{11} \mathrm{C}$ radionuclids and the background are present. (From Ref. [20]).

that the concentration of anomalous ${ }^{12} \tilde{\mathrm{C}}$ atoms in boron is $<5 \times 10^{-6} \mathrm{~g} / \mathrm{g}$. Since the cosmic abundance of carbon is $2 \times 10^{6}$ times greater than that of boron, this means that the relative concentration of anomalous carbon atoms in carbon corresponds to ${ }^{12} \tilde{\mathrm{C}} /{ }^{12} \mathrm{C}$ $<2.5 \times 10^{-12}$. Using this limit obtained, one finds from Eq.(1) that the lifetime of electrons in a carbon atom relative to the violation of the Pauli principle is $\tau>2 \times 10^{21}$ y.

In closing, note that the sensitivity of the $\gamma$-activation method, which was used to determine the ${ }^{12} \mathrm{C}$ concentration in boron, would in principle permit increasing the limit of the determination of $\tau$ by an order of magnitude if one could obtains a boron sample with a low content of "normal" carbon impurity or if the carbon could be effectively removed from the boron sample after irradiation.

\section{Testing PEP with the NEMO-2 detector [26]}

\subsection{NEMO-2 detector}

The NEMO-2 detector [34] was designed for double beta decay studies and operated in the Fréjus Underground Laboratory (4800 m w.e.) from 1991 to 1997. During this period, the two neutrino double beta decays of ${ }^{100} \mathrm{Mo}\left[35\right.$, ${ }^{116} \mathrm{Cd}\left[36\right.$ ], ${ }^{82} \mathrm{Se}$ [37] and 


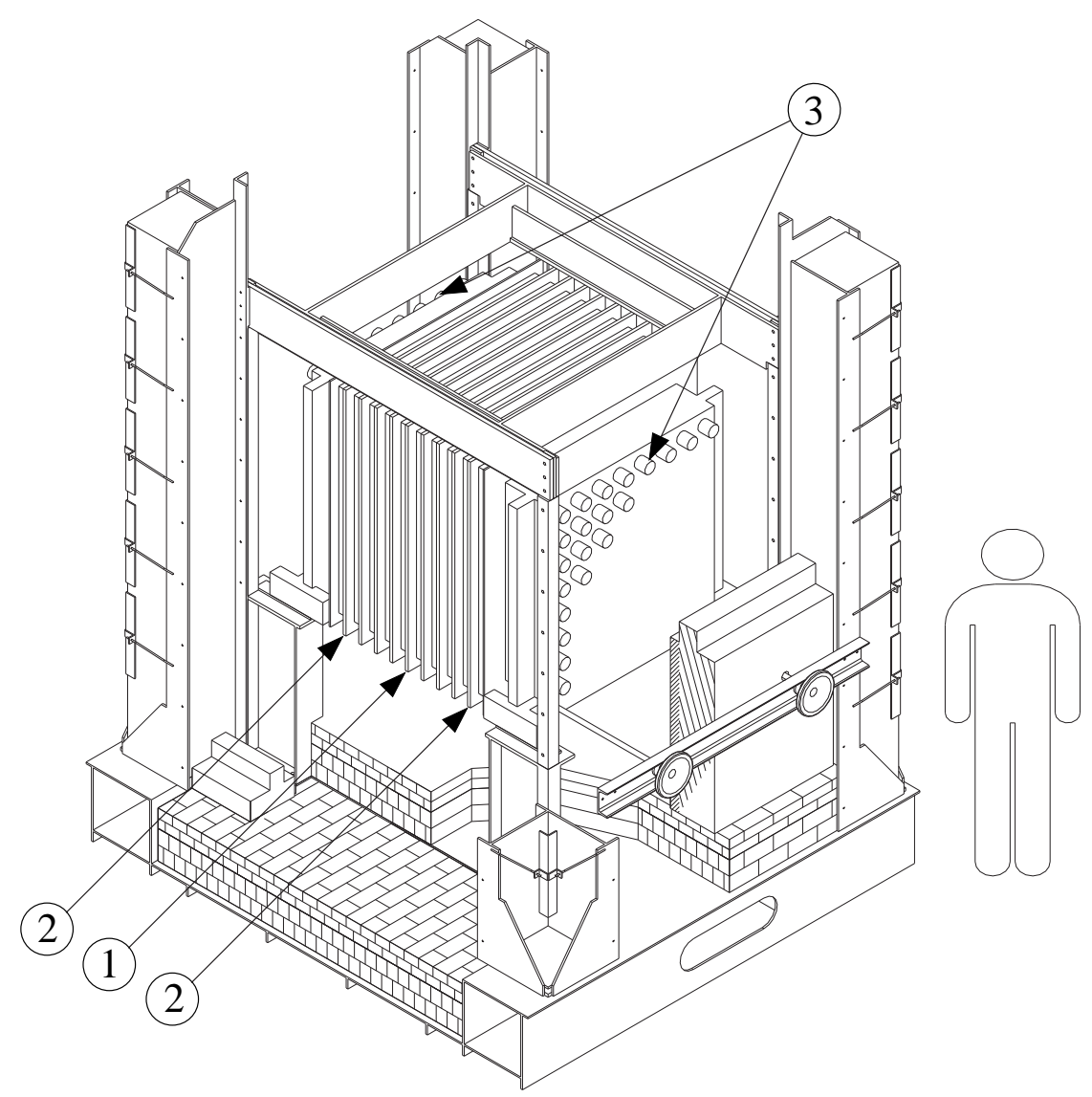

Figure 2. The NEMO-2 detector without shielding. (1) Central frame with the source plane was capable of supporting plural source foils. (2) The tracking device of 10 frames, each consisting of two perpendicular planes of 32 Geiger cells. (3) Two scintillator arrays each consisting of 5 by 5 counters for a calorimeter. In the earlier experiment with molybdenum sources 35] the scintillator arrays were 8 by 8 counters as depicted here.

${ }^{96} \mathrm{Zr} \mathrm{[38]} \mathrm{were} \mathrm{investigated} \mathrm{in} \mathrm{detail} \mathrm{through} \mathrm{the} \mathrm{measurements} \mathrm{of} \mathrm{the} \mathrm{summed} \mathrm{electron}$ energy spectra, angular distributions and single electron spectra.

The NEMO-2 detector (Fig. 2) consisted of a $1 \mathrm{~m}^{3}$ tracking volume filled with a mixture of helium gas and $4 \%$ ethyl alcohol. Vertically bisecting the detector was the plane of the source foil under study $(1 \mathrm{~m} \times 1 \mathrm{~m})$. Tracking was accomplished with long, open Geiger cells with an octagonal cross section defined by $100 \mu \mathrm{m}$ nickel wires. On each side of the source foil there were 10 planes of 32 cells which alternated between vertical and horizontal orientations.

A calorimeter made of scintillators covered two vertical opposing sides of the tracking volume. It consisted of two planes of 25 scintillators $(19 \mathrm{~cm} \times 19 \mathrm{~cm} \times 10 \mathrm{~cm})$, combined with low radioactivity photomultipliers tubes (PMT).

The tracking volume and scintillators were surrounded by a lead $(5 \mathrm{~cm})$ and iron $(20 \mathrm{~cm})$ shield for measurements with ${ }^{100} \mathrm{Mo}$ and ${ }^{116} \mathrm{Cd}$. The same shield was used in the experiment with ${ }^{82} \mathrm{Se}$ and ${ }^{96} \mathrm{Zr}$ foils for $6222.6 \mathrm{~h}$ with the $\mathrm{Zr}$ foils placed at the central 
part of the source plane. The lead was then placed outside the iron for $1784.5 \mathrm{~h}$. Next, the lead was removed for $536 \mathrm{~h}$. At the end, $15 \mathrm{~cm}$ of paraffin was installed outside of the iron for the final $2162.8 \mathrm{~h}$.

Details of the performance and parameters are described elsewhere [34, while the most salient characteristics are outlined briefly here. Three-dimensional measurements of charged particle tracks are provided by the array of Geiger cells. The transverse position is given by the drift time, and the longitudinal position is given by the plasma propagation times. The transverse resolution is $500 \mu \mathrm{m}$ and the longitudinal resolution is $4.7 \mathrm{~mm}$. The calorimeter's energy resolution (FWHM) is $18 \%$ at $1 \mathrm{MeV}$ with a time resolution of $275 \mathrm{ps}(550 \mathrm{ps}$ at $0.2 \mathrm{MeV})$. Scintillation counters measured the energy of an individual electron in the interval from $50 \mathrm{keV}$ to $4 \mathrm{MeV}$. Electrons with energies near or above $4 \mathrm{MeV}$ may fall into the "saturation" regime of the counters. More specifically, $E_{\text {sat }}$ is different for each counter and varies from 4 to $7 \mathrm{MeV}$. The only information for events in this regime is that they deposit an energy higher than $E_{\text {sat }}$. A laser and fiber optics device is used to check the stability of the scintillation detectors.

A trigger requiring one or two scintillation counters and four Geiger frames normally run at a rate of $0.01-0.04 \mathrm{~Hz}$ depending on the radon levels in the laboratory. This trigger rate is too low for an efficient calibration survey of the experiment, so a second trigger requiring only one counter with an energy greater than $1.3 \mathrm{MeV}$ was added.

An electron is defined by a track linking the source foil and one scintillator. The maximum scattering angle along the track has to be less than $20^{\circ}$ to reject hard scattering situations. A photon is recognized as one or two adjacent-fired scintillators, without an associated particle track. For photons and electrons, an energy deposited greater than $200 \mathrm{keV}$ is required in order to obtain sufficiently good time resolution for time-of-flight analysis of events. The two-electron events are defined by two tracks which have a common vertex in the source foil and are associated with two fired scintillators. A more detailed description can be found in the following references [34, 35, 36, 37].

\subsection{Experimental results}

The NEMO-2 detector's experimental data from measurements with $\mathrm{Cd}$, Se and $\mathrm{Zr}$ foils were used to estimate limits on non-Paulian transitions in the ${ }^{12} \mathrm{C}$ of the plastic scintilators. The total mass of ${ }^{12} \mathrm{C}$ under study was $170 \mathrm{~kg}$.

Fig. 3 shows non-Paulian transitions in ${ }^{12} \mathrm{C}$. In Fig. $3 \mathrm{a}$, the transition of a nucleon from the $p$-shell to the fully occupied $1 s_{1 / 2}$-shell is shown. This process is accompanied by $\gamma$-quantum emission, where its energy equals the energy difference between the $p$ and $s$ levels $(\sim 20 \mathrm{MeV})$ [27]. In subsequent figures, (Fig. 3b,c), the $\beta^{ \pm}$transitions of ${ }^{12} \mathrm{C}$ to non-Paulian ${ }^{12} \tilde{\mathrm{B}}$ and ${ }^{12} \tilde{\mathrm{N}}$ are shown when a nucleon falls from the $p$-shell to the fully occupied $1 s_{1 / 2}$-shell. The emitted $\beta^{+}$or $\beta^{-}$are distributed as ordinary $\beta$-decay spectra with an endpoint energy of $20 \mathrm{MeV}$ [28]. Cuts were used for extracting fine limits from the experiment. 

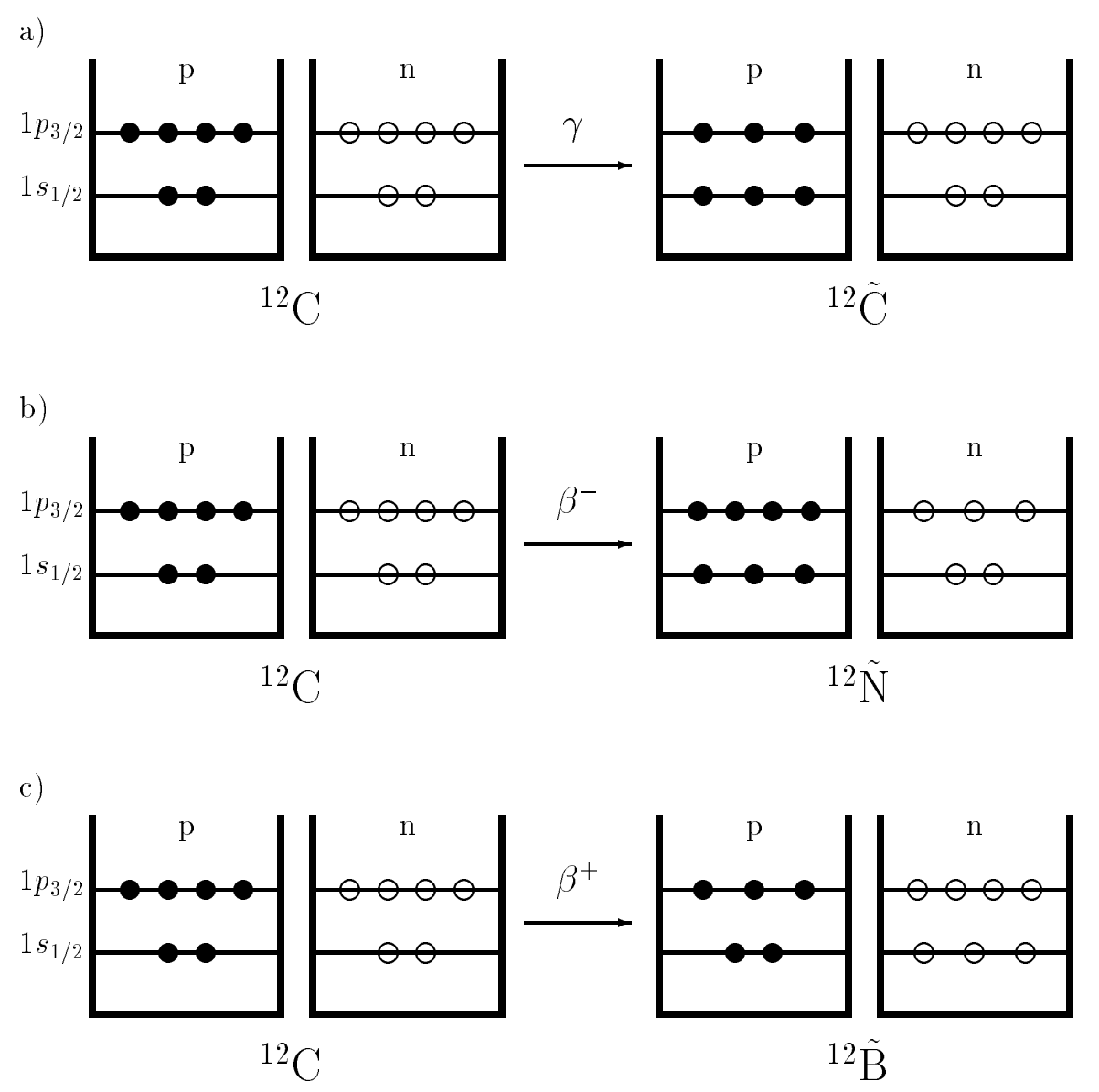

Figure 3. Schemes of non-Paulian transitions in ${ }^{12}$ C. (a) transition of a proton from the $p$-shell to the fully occupied $s$-shell (a similar figure can be constructed for neutrons) (b) non-Paulian $\beta^{-}$transition of ${ }^{12} \mathrm{C}$ to ${ }^{12} \tilde{\mathrm{N}}$; (c) non-Paulian $\beta^{+}$transition of ${ }^{12} \mathrm{C}$ to ${ }^{12} \tilde{\mathrm{B}}$.

3.2.1. Non-Paulian processes with high energy $\gamma$-quantum emission. High energy $\gamma$ quanta produced in a scintillator from the non-Paulian transition to ${ }^{12} \tilde{\mathrm{C}}$ were considered. The $\gamma$-quanta cross the tracking volume, interact with a source foil and give two tracks and two fired scintillators. In the energy region $E_{\gamma} \sim 20 \mathrm{MeV}$, pair creation probability in the source foil $\left(45-50 \mathrm{mg} / \mathrm{cm}^{2}\right)$, is higher by 2-3 orders of magnitude than those for double Compton interactions or Möller scattering of Compton electrons in the foil. The NEMO-2 detector was not designed to distinguish between $e^{+}$and $e^{-}$tracks, thus pairs were detected as two electron events (2e). A time-of-flight analysis was used to select high energy $2 \mathrm{e}$ events in both simulation and experimental data. The simulated data studied $3.8 \cdot 10^{6}$ events with initial $\gamma$-quanta emitted from the scintillators. The maximum in the summed electron energy $\left(E_{2 \mathrm{e}}\right)$ spectrum (Fig. 4$)$ is at the energy of the $\gamma$-quanta minus the two electron's masses.

No events with two tracks and summed energy $\geq 4 \mathrm{MeV}$ were found in the experiment with Se and Zr given an exposure of $10357 \mathrm{~h} \mathrm{[37,} \mathrm{38]} \mathrm{and} \mathrm{in} \mathrm{the} \mathrm{enriched} \mathrm{Cd}$

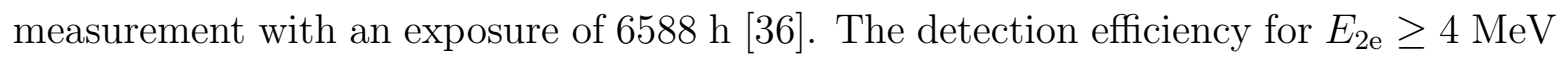




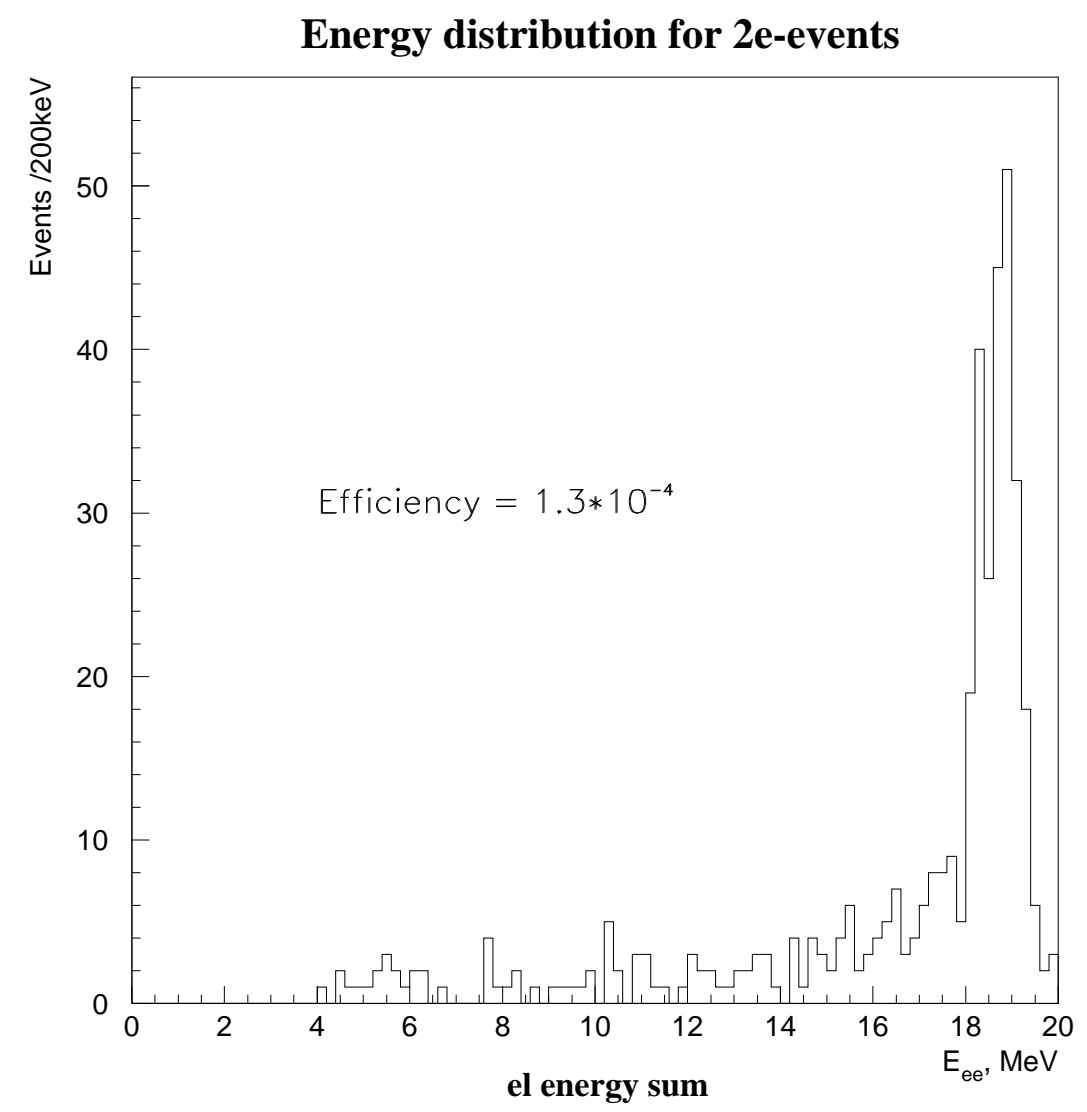

Figure 4. Simulated summed electron energy spectrum of two-electron events coming from the source foil for which the event was generated by a $20 \mathrm{MeV} \gamma$-quanta in the plastic scintillators. A low energy cut is applied at $4 \mathrm{MeV}$. (From Ref. [26]).

and $\cos \left(\theta_{2 \mathrm{e}}\right)>0$ is equal to $0.013 \%$ for the $\mathrm{Se}$ and $\mathrm{Zr}$ sources. In the case of the enriched $\mathrm{Cd}$ the efficiency should be scaled by a factor of 0.57 because the enriched $\mathrm{Cd}$ is another material with a different thickness and occupied only a half of the source plane. From the data one can obtain a limit on the PEP-violated transition of ${ }^{12} \mathrm{C}$ nucleus to ${ }^{12} \tilde{\mathrm{C}}$ at the $90 \%$ C.L. of:

$$
T_{1 / 2}>5.3 \cdot 10^{23} y
$$

Also the limit on PEP violating transitions of nucleons from the $p$-shell to the fully occupied $1 s_{1 / 2}$-shell in ${ }^{12} \mathrm{C}$ at the $90 \%$ C.L. is:

$$
T_{1 / 2}>4.2 \cdot 10^{24} y
$$

3.2.2. $\beta^{ \pm}$decays to non-Paulian states. The search for $\beta^{ \pm}$decay processes was performed through the selection of two track events. Cuts for these events require an electron to appear in a plastic scintillator, cross the tracking volume and source plane then enter a plastic scintillator on the opposite side of the NEMO-2 detector. Simulations of $\beta^{+}$and $\beta^{-}$decays of ${ }^{12} \mathrm{C}$ to non-Paulian states of the daughter nuclei 


\section{Energy distribution for 2e-events}
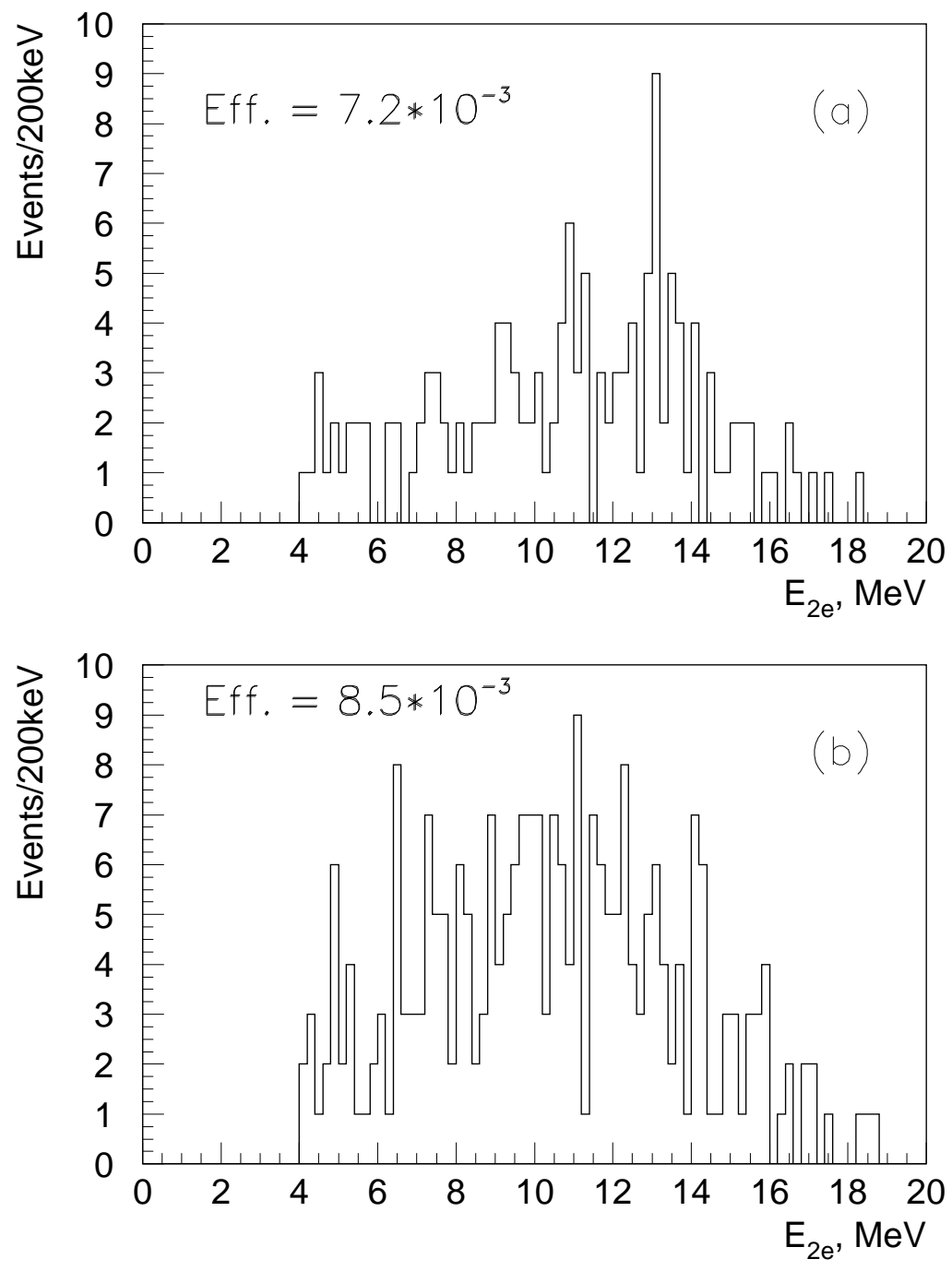

Figure 5. Energy spectra of simulated two track events: (a) for $\beta^{+}$decay and (b) for $\beta^{-}$decay of ${ }^{12} \mathrm{C}$ to non-Paulian states of daughter nuclei with a $20 \mathrm{MeV}$ endpoint energy. Here again there is a cut at $4 \mathrm{MeV}$ for comparison with the experimental data. (From Ref. [26]).

in plastic scintillators was examined. The simulated spectra are presented in Fig. 5. Evident here is that the efficiency for $\beta^{+}$decays is lower than $\beta^{-}$decays because the detection of at least one annihilation $\gamma$-quantum (511 keV) leads to the rejection of such an event.

The main background in the energy range up to $8 \mathrm{MeV}$ is due to neutrons from natural sources. Consequently, the data used here was obtained in a run with a paraffin shield $(2162.8 \mathrm{~h})$, which efficiently suppressed the neutron background. Only one event with a summed energy deposit of $E>4 \mathrm{MeV}$ was found in the experiment involving Se 
Table 1. Limits on the non-Paulian transitions in ${ }^{12} \mathrm{C}$. (From Ref. [26]).

\begin{tabular}{llll}
\hline Channel & $\gamma$ emission & $\beta^{-}$decay & $\beta^{+}$decay \\
\hline Window $(\mathrm{MeV})$ & {$[4,20]$} & {$[4,20]$} & {$[4,20]$} \\
Number of events & 0 & 1 & 1 \\
Efficiency & $1.3 \cdot 10^{-4}$ & $8.5 \cdot 10^{-3}$ & $7.2 \cdot 10^{-3}$ \\
$T_{1 / 2}(90 \% \mathrm{CL})$ present & $>4.2 \cdot 10^{24} \mathrm{y}$ & $>3.1 \cdot 10^{24} \mathrm{y}$ & $>2.6 \cdot 10^{24} \mathrm{y}$ \\
\hline$T_{1 / 2}(99.7 \% \mathrm{CL})[27]$ & $>1.3 \cdot 10^{20} \mathrm{y}$ & \\
$T_{1 / 2}(90 \% \mathrm{CL})[28]$ & & $>8 \cdot 10^{27} \mathrm{y}$ & $>8 \cdot 10^{27} \mathrm{y}$ \\
\hline
\end{tabular}

and $\mathrm{Zr}$ samples. The detection efficiency of $\beta^{-}$is equal to $0.85 \%$, and for $\beta^{+}$is $0.72 \%$. As a result, one can obtain limits on $\beta^{ \pm}$decays of ${ }^{12} \mathrm{C}$ to non-Paulian states at the $90 \%$ C.L.:

$$
T_{1 / 2}>3.1 \cdot 10^{24} y \quad \text { for } \beta^{-}
$$

and

$$
T_{1 / 2}>2.6 \cdot 10^{24} y \quad \text { for } \beta^{+} .
$$

\subsection{Conclusion and prospects for the future}

Table 1 presents the NEMO-2 results on non-Paulian transitions in ${ }^{12} \mathrm{C}$. Due to good time-of-flight selection and a large mass of plastic scintillator the limits for $20 \mathrm{MeV}$ gamma emission is higher by four orders of magnitude than the previous limit [27]. Limits on the $\beta^{ \pm}$non-Paulian transitions are lower than in [28], because of the relatively small masses involved and the low efficiency for crossing electron detection. Recently the limit on the transition with the emission of a $\gamma$-quantum was improved by BOREXINO to $T_{1 / 2}>2.1 \cdot 10^{27}$ y [30].

The new detector, NEMO-3, which is functioning [39, 40], will improve these limits. The amount of ${ }^{12} \mathrm{C}$ is $\sim 40$ times greater and the detection efficiency $\sim 10$ times higher. Additionally a magnetic field is applied to distinguish $e^{+} e^{-}$from $e^{-} e^{-}$events. So expected limits which will be obtained with the NEMO-3 detector will be three orders of magnitude higher. The sensitivity of the next generation experiment (SuperNEMO [41, 42]) can be estimated to be $\sim 10^{28}-10^{29}$ y for all processes mentioned above.

\section{Statistic of neutrinos and double beta decay [32]}

Do neutrinos respect the exclusion principle of its inventor? In this paper it is assumed that the Pauli exclusion principle is violated for neutrinos and therefore neutrinos obey (at least partly) Bose-Einstein statistics.

It may happen that due to unique properties of neutrinos, a violation of the Pauli principle in the neutrino sector might be much stronger than in other particle sectors. Therefore the effects of its violation may first be seen in neutrino physics. 
A possibility of Bose statistics for neutrinos was first considered in ref. [43] where its effects on big bang nucleosynthesis (BBN) have been studied. According to [43] the change of neutrino statistics from pure fermionic to pure bosonic diminishes the primordial ${ }^{4} \mathrm{He}$ abundance by $\sim 4 \%$.

The idea of bosonic neutrinos has been proposed independently in ref. [44, where cosmological and astrophysical consequences of this hypothesis have been studied. Bosonic neutrinos might form a cosmological Bose condensate which could account for all, or a part of, the dark matter in the universe. "Wrong" statistics of neutrinos modifies BBN, leading to the effective number of neutrino species being smaller than three. The conclusion in [44] agrees qualitatively with the results of [43], though quantitatively a smaller decrease of $N_{\nu}$ is found [45].

As far as astrophysical consequences are concerned, dynamics of the supernova collapse would be influenced and spectra of a supernova neutrinos may change [44, 46]. The presence of neutrino condensate would enhance contributions of the Z-bursts to the flux of the ultra high energy (UHE) cosmic rays and lead to substantial refraction effects for neutrinos from remote sources [44].

A violation of the Pauli principle for neutrinos should show up in the elementary processes where identical neutrinos are involved. A realistic process for this test is the two-neutrino double beta decay. It was shown in [44] that the probability of the decay, as well as the energy spectrum and angular distribution of the electrons should be affected. Qualitative conclusions were that the pure bosonic neutrino is excluded, whereas a large fraction of the bosonic component in a neutrino state is still allowed by the present data. In this connection, a possibility of partly bosonic neutrinos should be considered.

\subsection{Result of calculations}

In the case of ${ }^{100} \mathrm{Mo}$ the decay proceeds mainly through the $1^{+}$intermediate nucleus and the single state dominance (SSD) hypothesis should give a good approximation. This is also confirmed by spectra measurements in the NEMO-3 experiment [47, 48].

Using the SSD approximation one can calculate the $2 \nu \beta \beta$-decay half-life of ${ }^{100} \mathrm{Mo}$ to the ground state for fermionic [49] and bosonic neutrinos

$$
T_{1 / 2}^{f}\left(0_{\text {g.s. }}^{+}\right)=6.810^{18} \text { years, } \quad T_{1 / 2}^{b}\left(0_{\text {g.s. }}^{+}\right)=8.910^{19} \text { years, }
$$

so that the ratio of probabilities equals

$$
r_{0}\left(0_{\text {g.s. }}^{+}\right)=0.076
$$

The ratio $r_{0}\left(0_{\text {g.s. }}^{+}\right)$determines the weight with which the bosonic component enters the total rate and differential distribution.

The higher intermediate levels can give some (basically unknown) contribution and this produces a systematic error in the analysis. To evaluate the effect of the higher states, one can consider the extreme case described by the higher states dominance 


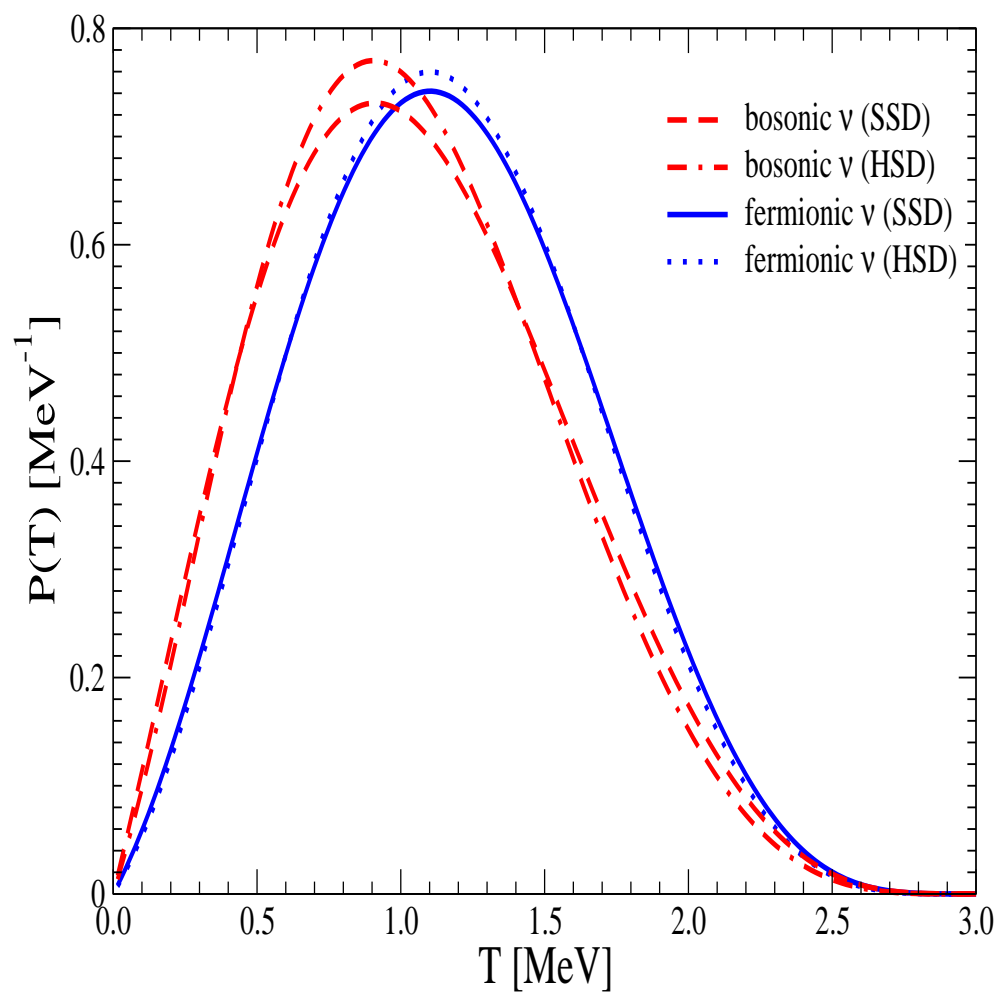

Figure 6. The differential decay rates normalized to the total decay rate vs. the sum of the kinetic energy of outgoing electrons $\mathrm{T}$ for $2 \nu \beta \beta$-decay of ${ }^{100} \mathrm{Mo}$ to the ground state of the final nucleus. The results are presented for the cases of pure fermionic and bosonic neutrinos. The calculations have been performed within the single-state dominance hypothesis (SSD) and with the assumption of dominance of higher lying states (HSD). (From Ref. [32]).

(HSD) approximation, which allows one to factorize the nuclear matrix element and integration over the phase space of outgoing leptons.

The energy spectra of electrons calculated in the SSD and HSD approximations are presented in the Figs. 6 and 7. The SSD approximation gives a slightly wider spectra of two electrons for both the fermionic and bosonic neutrinos. The spectra for the bosonic neutrinos are softer in both approximations. In particular, the maxima of SSD and HSD spectra are shifted to low energies for bosonic neutrinos by about $15 \%$ with respect to fermionic-neutrino spectra. This shift does not depend on the approximation and therefore, can be considered as the solid signature of the bosonic neutrino. Also the energy spectrum for single electrons becomes softer in the bosonic case (Fig. 17).

In Fig. 8 the two electron energy spectra for different values of the bosonic-fraction $\sin ^{2} \chi$ show the shift to smaller energies with increasing $\sin ^{2} \chi$. Due to the smallness of $r_{0}$, substantial shifts occur only when $\sin ^{2} \chi$ is close to 1.0. The probability of $2 \nu \beta \beta$ decay is then equal to:

$$
W_{t o t}=\cos ^{4} \chi W_{f}+\sin ^{4} \chi W_{b}
$$

Fig. 9) shows the energy spectra of single electrons for different values of $\sin ^{2} \chi$. Note 


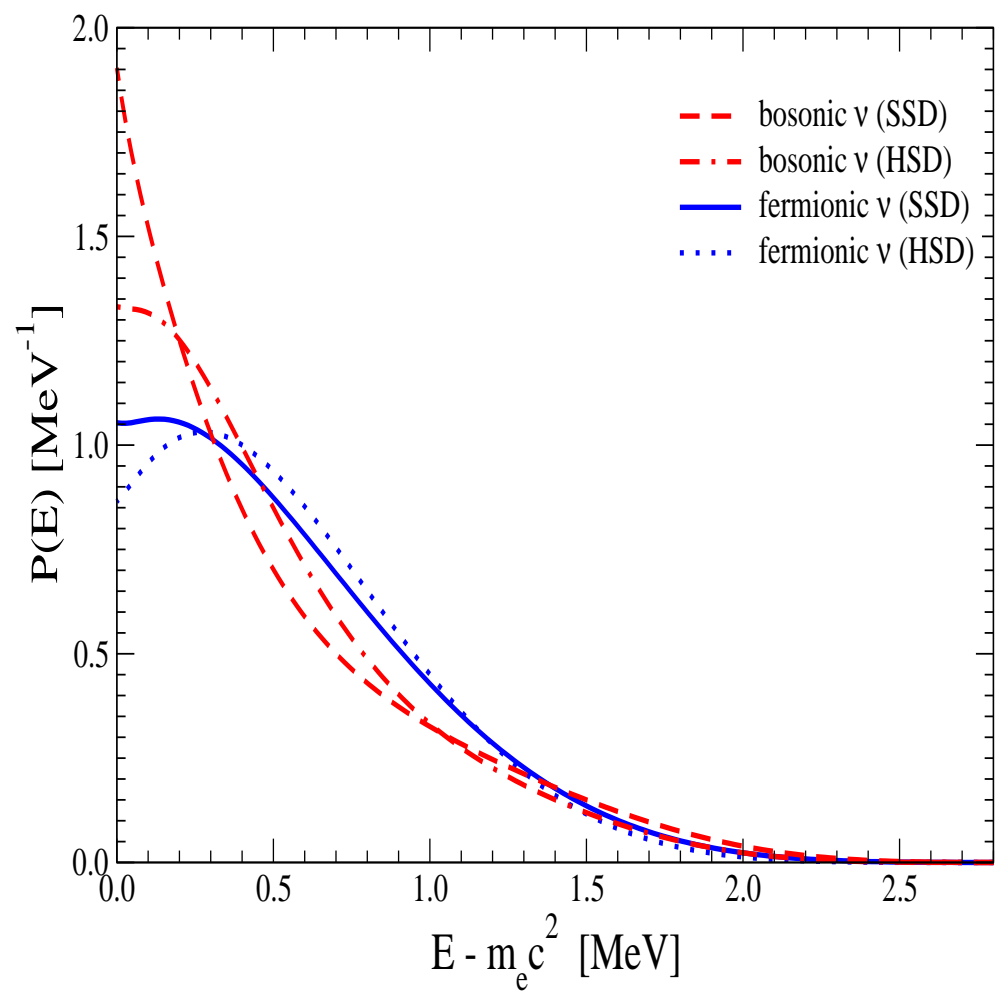

Figure 7. The single electron differential decay rate normalized to the total decay rate vs. the electron energy for $2 \nu \beta \beta$-decay of ${ }^{100}$ Mo to the ground state of the final nucleus. $E$ and $m_{e}$ represent the energy and mass of the electron. The results are presented for the cases of pure fermionic and bosonic neutrinos. (From Ref. [32]).

a substantial change occurs at very low energies, with $E=0.3 \mathrm{MeV}$ being a fixed point. For $E<0.3 \mathrm{MeV}$ the distribution increases with $\sin ^{2} \chi$, whereas for $E=0.3-1.4 \mathrm{MeV}$ it decreases.

\subsection{Bounds on bosonic neutrinos}

Measurements of the differential characteristics of the decays should show different shapes of the single and summed electron energies as well as the angular distribution. Such information is provided now by NEMO-3 for ${ }^{100} \mathrm{Mo},{ }^{82} \mathrm{Se},{ }^{116} \mathrm{Cd},{ }^{150} \mathrm{Nd},{ }^{96} \mathrm{Zr}$, ${ }^{48} \mathrm{Ca}$ and ${ }^{130} \mathrm{Te}$ [40, 47, 48, 50]. One should perform the statistical fit of the spectra by a general distribution with $\sin ^{2} \chi$ being a free parameter. The spectral method is sensitive to $\sin ^{2} \chi$ for nuclei and transitions with large $r_{0}$. That includes ${ }^{100} \mathrm{Mo}$, as well as transitions to the excited states.

First consider the energy spectra of $0_{\text {g.s. }}^{+} \rightarrow 0_{\text {g.s. }}^{+}$decay of ${ }^{100} \mathrm{Mo}$ [40]. In the present paper no detailed statistical analysis of the spectra is applied at this time, postponing this to the time when the measurements are finished and there are careful calibrations. Instead, there are some qualitative estimates. Here there is reasonable agreement 


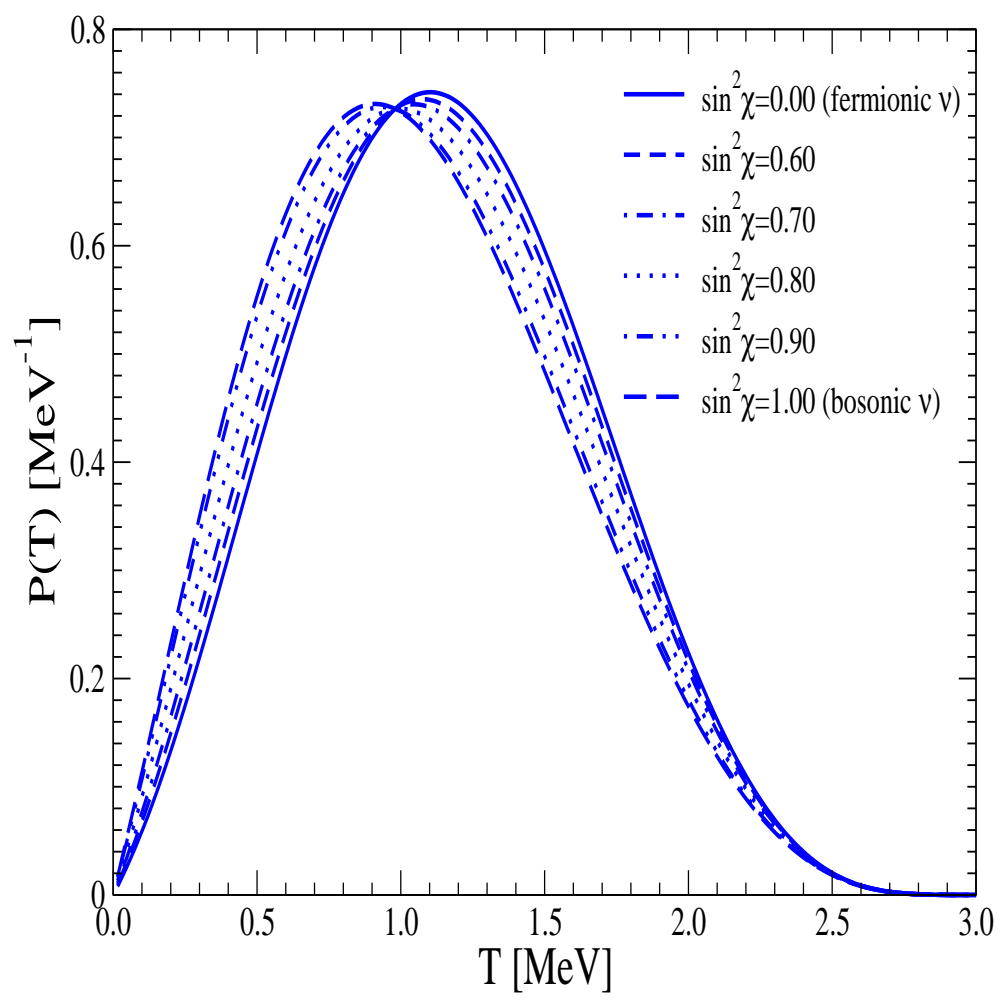

Figure 8. The differential decay rates normalized to the total decay rate vs. the sum of the kinetic energy of outgoing electrons (T) for $2 \nu \beta \beta$-decay of ${ }^{100} \mathrm{Mo}$ to the ground state of the final nucleus. The results are presented for different values of the squared admixture of the bosonic component $\left(\sin ^{2} \chi\right)$. The spectra have been calculated in the SSD approximation. (From Ref. [32]).

with the predicted energy spectrum of the two electrons and the experimental points. Therefore one can certainly exclude the pure bosonic case $\left(\sin ^{2} \chi=1\right)$. Furthermore, comparing in Fig. 8 the relative shift of the bosonic maximum with the experimental spectrum one can put the conservative bound on $\sin ^{2} \chi<0.6$. In fact, there is no ideal agreement between the data and theoretical spectrum. A better fit can be obtained for $\sin ^{2} \chi$ between 0.4 and 0.5 .

Next a comment is made on the single-electron energy spectrum from ${ }^{100} \mathrm{Mo}$ decay. The data agrees well with the predictions from the fermionic SSD mechanism, but some difference exists between the data and the fermionic HSD mechanism predictions. From this it was concluded that the SSD mechanism is better here [47, 48]. Comparing the experimental data and spectra for partly bosonic neutrinos (Fig. 9) one obtains $\sin ^{2} \chi<0.7$.

Notice that the SSD spectrum does not show an ideal agreement with the experimental data either. There is a discrepancy in the low energy region $(E=0.2-0.4$ $\mathrm{MeV})$. That could be explained by the effect of partly bosonic neutrinos with $\sin ^{2} \chi \sim$ $0.5-0.6$. 


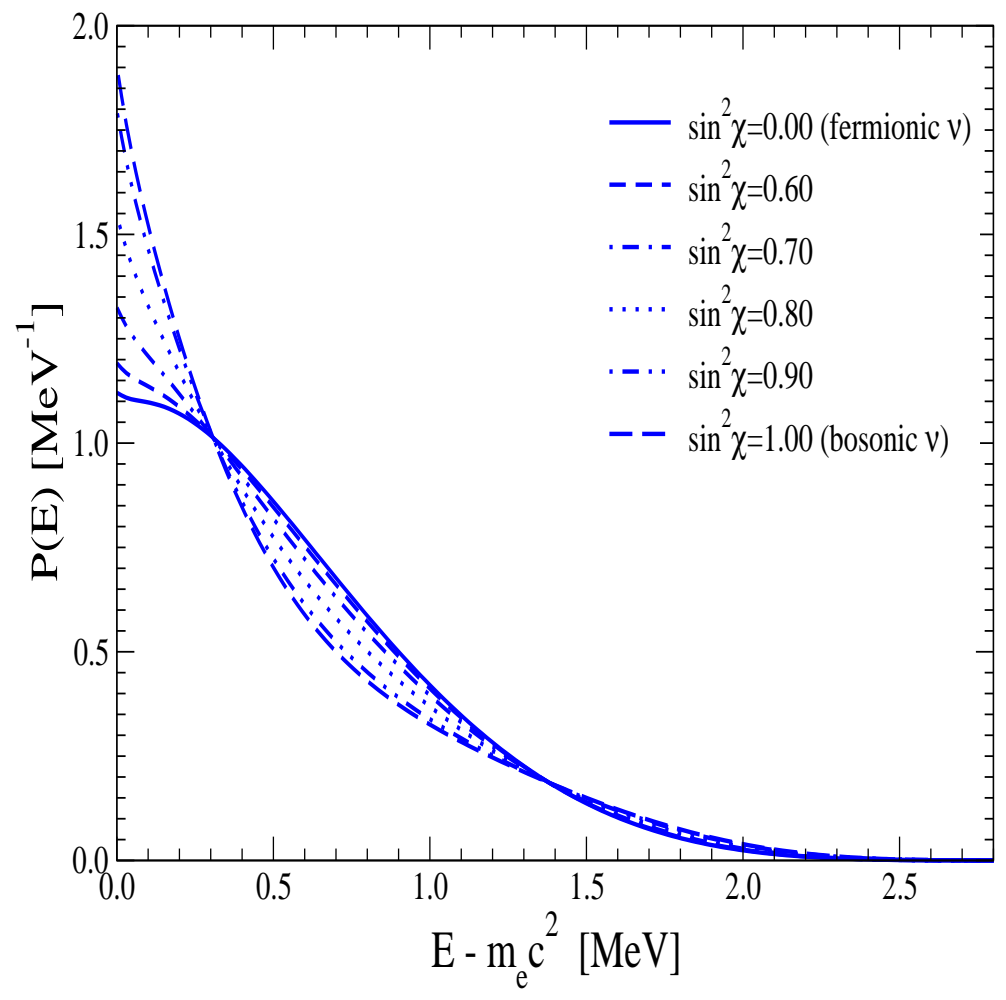

Figure 9. The single electron differential decay rate normalized to the total decay rate vs. the electron energy for $2 \nu \beta \beta$-decay of ${ }^{100} \mathrm{Mo}$ to the ground state of the final nucleus. The results are presented for different values of the squared admixture of the bosonic component $\left(\sin ^{2} \chi\right)$. The spectra have been calculated in the SSD approximation. The conventions are the same as in Fig. 7. (From ref. 32]).

The full analysis of existing NEMO-3 information (energy and angular distributions) using maximal likelihood methods, will have a higher sensitivity to $\sin ^{2} \chi$. However, it is difficult to expect a better bound than $\sin ^{2} \chi \sim 0.4-0.5$, because of the existing disagreement between the data and Monte Carlo (MC) simulations. In fact, it can be just some systematic effect connected to the present poor understanding of the response function of the detector. If in the future the NEMO experimental data turns out to be in better agreement with the MC-simulated spectrum, the sensitivity to the partly bosonic neutrino will be improved down to $\sin ^{2} \chi \sim 0.2-0.3$.

Finally the determination of the ratios of half-lives to the excited and ground state is,

$$
r_{f, b}^{*}\left(J^{\pi}\right) \equiv \frac{T_{1 / 2}^{f, b}\left(J^{\pi}\right)}{T_{1 / 2}^{f, b}\left(0_{g . s .}^{+}\right)},
$$

for the fermionic and bosonic neutrinos. For $2 \nu \beta \beta$-decay of ${ }^{100}$ Mo the ratio can be calculated rather reliably using the SSD approximation. The advantage of this quantity is that the EC amplitude, [(A,Z) $\rightarrow(\mathrm{A}, \mathrm{Z}+1)$ transition], which is not well determined, cancels in the ratio (5). 
For ${ }^{100}$ Mo the transitions to the ground $\left(0_{\text {g.s. }}^{+}\right)$and excited $\left(0_{1}^{+}\right)$states have been detected, and a discrepancy has been observed. The corresponding experimental ratio $r^{*}$ equals

$$
r_{\text {exp. }}^{*}\left(0_{1}^{+}\right) \simeq 80
$$

(NEMO-3 results [40, 51]), whereas within the SSD approach the calculated ones are

$$
\begin{aligned}
r^{*}\left(0_{1}^{+}\right) & \simeq 61 \quad(\text { fermionic } \nu) \\
& \simeq 73 \quad(\text { bosonic } \nu) .
\end{aligned}
$$

The bosonic neutrino fits the data slightly better but the differences are probably beyond the accuracy of the SSD assumption. Nevertheless, it is also possible to improve the statistics in the measurements of the transition to the excited $0_{1}^{+}$state.

Contrary to the case of the $0^{+}$excited state, the ratio of $2 \nu \beta \beta$-decay half-lives to the excited $2^{+}$and ground state is expected to be strongly different for the bosonic and

fermionic neutrinos. Using the SSD approximation for the $2 \nu \beta \beta$-decay of ${ }^{100} \mathrm{Mo}$ these are

$$
\begin{aligned}
r^{*}\left(2_{1}^{+}\right) & \simeq 2.510^{4} \quad & & (\text { fermionic } \nu) \\
& \simeq 2.710^{2} \quad & & (\text { bosonic } \nu) .
\end{aligned}
$$

The $2 \nu \beta \beta$-decay of ${ }^{100}$ Mo to the excited $2_{1}^{+}$state has not been measured yet. Using the best experimental limit on the half-life found in [52] one gets

$$
r_{\text {exp }}^{*}\left(2_{1}^{+}\right)>2.210^{2} \text {. }
$$

This bound is close to the bosonic prediction. Further experimental work in measuring this nuclear transition will allow one to analyze the case of the partially bosonic neutrino.

\section{Conclusion}

A search was made for anomalous carbon atoms $\left({ }^{12} \tilde{\mathrm{C}}\right)$, with three K-shell electrons. A limit on the existence of such atoms was determined that is ${ }^{12} \tilde{\mathrm{C}} /{ }^{12} \mathrm{C}<2.5 \times 10^{-12}$. This corresponds to a lifetime limit with respect to the violation of the Pauli principle by electrons in a carbon atom of $\tau>2 \times 10^{21} \mathrm{y}$.

PEP was tested with the NEMO-2 detector. In the future using NEMO-3 and SuperNEMO the sensitivity can be increased to $\sim 10^{28}-10^{29} \mathrm{y}$.

This was the first time PEP was checked for neutrinos. Here pure bosonic neutrinos are excluded by the present $\beta \beta$ decay data. In the case of partly bosonic neutrinos the analysis of the existing data allows one to put the upper bound on $\sin ^{2} \chi$ of $<0$. The sensitivity can be improved up to $\sin ^{2} \chi \sim 0.1-0.2$.

\section{Acknowledgments}

I am very thankful to Prof. S. Sutton for his useful remarks. Portions of this work were supported by a grant from RFBR (no 06-02-72553). This work was supported by Russian Federal Agency for Atomic Energy. 


\section{References}

[1] Pauli W., Z.Phys. 31, 765 (1925).

[2] Wu C.S., Ambler E., Hayward R.W. et al., Phys. Rev. 105, 1413 (1957).

[3] Christinsen J.H., Cronin J.W., Fitch V.L. et al., Phys. Rev. Lett. 13, 138 (1964).

[4] Okun L.B., Zeldovich Ya.B., Phys. Lett. B 78, 597 (1978).

[5] Ignatiev A.Yu., Kuzmin V.A., Shaposhnikov M.E., Phys. Lett. B 84, 315 (1979).

[6] Voloshin M.B., Okun L.B., JETP Lett. 28, 145 (1978).

[7] Mohapatra R.N., Nussinov S., J.Mod.Phys. A 7, 3817 (1992).

[8] Ellis J., Mavromatos N.E., Nanopoulos D.V., Proc. Int. Workshop on K Physics, Ed. Lydia Iconomidou-Fayard (Editions Frontieres, Orsay, France, 1997) 187.

[9] Kostelecky V.A., ibid, 407.

[10] Nielsen H.B., Ninomiya M., Nucl. Phys. B 141, 153 (1978).

[11] Kostelecky V.A., Samuel S., Phys. Rev. D 39, 683 (1989).

[12] Ignatiev A.Yu., V.A. Kuzmin V.A., Sov. Journ. Nucl. Phys. 56, 444 (1987).

[13] Okun L.B., JETP Lett. 46, 529 (1987).

[14] Greenberg O.W., Mohapatra R.N., Phys. Rev. Lett. 59, 2507 (1987).

[15] Greenberg O.W., Mohapatra R.N., Phys. Rev. D 39, 2032 (1989).

[16] Greenberg O.W., Mohapatra R.N., Phys. Rev. Lett. 62, 712 (1989).

[17] Okun L.B., Sov. Phys. Usp. 32, 543 (1989).

[18] Govorkov A.B., Phys. Lett. A 137, 7 (1989).

[19] Govorkov A.B., preprint JINR E2-90-364 (1990).

[20] Barabash A.S. et al., JETP Lett. 68, 112 (1998).

[21] Novikov V.M. et al., Phys. Lett. B 240, 227 (1990).

[22] Javorsek II D. et al., Phys. Rev. Lett. 85, 2701 (2000).

[23] Bartalucci S. et al., Phys. Lett. B 641, 18 (2006).

[24] Curceanu C. et al., quant-ph/0803.0870.

[25] Ignatiev A.Yu., Rad. Phys. Chem. 75, 2090 (2006).

[26] Arnold R. et al., Eur. J. Phys. A 6, 361 (1999).

[27] Logan B.A., Ljubicic A., Phys. Rev. C 20, 1957 (1979).

[28] Kekez D., Ljubicic A., Logan B.A., Nature 348, 224 (1990).

[29] Baron E, Mohapatra R.N. and Teplitz V.L., Phys. Rev. D 59, 036003 (1999).

[30] Back H.O. et al., Eur. Phys. J. C 37, 421 (2004).

[31] Bernabey R. et al., Phys. Lett. B 408, 439 (1997).

[32] Barabash A.S. et al., Nucl. Phys. B 783, 90 (2007).

[33] Novikov V.M., Pomansky A.A., JETP Lett. 49, 81 (1989).

[34] Arnold R. et al., Nucl. Instr. Meth. A 354, 338 (1995).

[35] Dassie D. et al., Phys. Rev. D 51, 2090 (1995).

[36] Arnold R. et al., Z. Phys. C 72, 239 (1996).

[37] Arnold R. et al., Nucl. Phys. A 636, 209 (1998).

[38] Arnold R. et al., Nucl. Phys. A 658, 299 (1999).

[39] Arnold R. et al., Nucl. Instr. Meth. A 536, 79 (2005).

[40] Arnold R. et al., Phys. Rev. Lett. 95, 182302 (2005).

[41] Barabash A.S., Czech. J. Phys. 52, 575 (2002).

[42] Ohsumi H., J. Phys.: Conf. Ser. 120, 052054 (2008).

[43] Cucurull L., Grifols J.A., Toldra R., Astropart. Phys. 4, 391 (1996).

[44] Dolgov A.D., Smirnov A.Yu., Phys. Lett. B 621, 1 (2005).

[45] Dolgov A.D., Hansen S.H., Smirnov A.Yu., JCAP 0506, 004 (2005).

[46] Choubey S., Kar K., Phys. Lett. B 634, 14 (2006).

[47] Arnold R. et al. JETP Lett. 80, 377 (2004).

[48] The NEMO Collaboration (Shitov Yu. et al.) Phys. At. Nucl. 69, 2090 (2006). 
[49] Domin P., Kovalenko S., Simkovic F., Semenov S.V., Nucl. Phys. A 753, 337 (2005).

[50] Barabash A.S., hep-ex 0807.2948 (2008).

[51] Arnold R. et al., Nucl. Phys. A 781, 209 (2007).

[52] Barabash A. et al., Phys. Lett. B 345, 408 (1995). 